\title{
Prehospital exenatide in hyperglycemic stroke-A randomized trial
}

\section{Larsson, Martin}

2019-12

Larsson, M , Castren, M , Lindström , V , von Euler , M, Patrone , C , Wahlgren , N \&

Nathanson , D 2019 , ' Prehospital exenatide in hyperglycemic stroke-A randomized trial ', Acta Neurologica Scandinavica , vol. 140 , no. 6 , pp. 443-448 . https://doi.org/10.1111/ane.13166

http://hdl.handle.net/10138/312965

https://doi.org/10.1111/ane.13166

acceptedVersion

Downloaded from Helda, University of Helsinki institutional repository.

This is an electronic reprint of the original article.

This reprint may differ from the original in pagination and typographic detail.

Please cite the original version. 
DR. MARTIN LARSSON (Orcid ID : 0000-0002-3226-7397)

Article type : Original Article

\section{Prehospital exenatide in hyperglycemic stroke - a randomized trial}

Running title: Prehospital exenatide in hyperglycemic stroke

Martin Larsson ${ }^{1}$, Maaret Castren ${ }^{1,2}$, Veronica Lindström ${ }^{3,4}$, Mia von Euler $^{1,5}$, Cesare Patrone ${ }^{1}$, Nils Wahlgren ${ }^{6}$, David Nathanson ${ }^{1}$

${ }^{1}$ Department of Clinical Science and Education at Södersjukhuset, Karolinska Institutet Department of Internal Medicine, Södersjukhuset,

${ }^{2}$ Department of Emergency Medicine and Services, Helsinki University Hospital, Finland

${ }^{3}$ Department of Neurobiology, Care Sciences and Society, section of nursing, Karolinska Institutet.

${ }^{4}$ Academic Emergency Medical Service, Region Stockholm, Sweden.

${ }^{5}$ Depatment of Medicine Solna, Stroke Research Network, Karolinska Institutet, Stockholm, Sweden

${ }^{6}$ Depatment of Clinical Neuroscience, Karolinska Institutet, Stockholm, Sweden

Corresponding author: Martin Larsson, Internal Medicine, Södersjukhuset, Department of Clinical Science and Education at Södersjukhuset, Karolinska Institutet, Sjukhusbacken 10, 11883 Stockholm, Sweden. Email: martin.larsson.1@ki.se

\section{Ackowledgements}

The author(s) disclosed receipt of the following financial support for the research, authorship, and/or publication of this article: The Swedish Heart and Lung Foundation as

This article has been accepted for publication and undergone full peer review but has not been through the copyediting, typesetting, pagination and proofreading process, which may lead to differences between this version and the Version of Record. Please cite this article as doi: 10.1111/ane.13166

This article is protected by copyright. All rights reserved 
part of Mission Fight Stroke, European Foundation for the Study of Diabetes, the Swedish Research Council, O. E. och Edla Johanssons Stiftelse and by STROKE Riksförbundet. Roche Diagnostics provided a grant and the glucometers Accu-Chek ${ }^{\circledR}$ Inform II.

\section{Declarations}

\section{Conflicting Interests}

The authors declare that there is no conflict of interest.

\section{Ethical approval}

The study was approved by the Central Ethics Committee of Sweden (Ref. No. Ö 6-2012). Written informed consent was obtained from all participants before entry.

\section{Contributorship}

ML, DN and MC designed the study. ML, VL and DN conducted the study. ML collected and analyzed the data and wrote the manuscript. ML, MC, MvE, CP, VL, NW and DN interpreted the data and edited the manuscript. All authors approved the final version of the manuscript.

\section{Data availability}

The data that support the findings of this study are available from the corresponding author upon reasonable request.

\section{Abstract}

Objectives: Hyperglycemia is a predictor for poor stroke outcome. Hyperglycemic stroke patients treated with thrombolysis have an increased risk of intracranial hemorrhage. Insulin is the gold standard for treating hyperglycemia but comes with a risk of hypoglycemia. Glucagon like peptide-1 receptor agonists (GLP-1RA) are drugs used in type 2 diabetes that have a low risk of hypoglycemia and have been shown to exert neuroprotective effects. The primary objective was to determine whether prehospital administration of the GLP-1RA exenatide could lower plasma glucose in stroke patients. Secondary objective was to study tolerability and safety. 
Materials \& Methods: Randomized controlled trial comparing exenatide administrated prehospitally with a control group receiving standard care for hyperglycemia. Patients with Face Arm Speech Test $\geq 1$ and glucose $\geq 8 \mathrm{mmol} / \mathrm{L}$ were randomized. Glucose was monitored for 24 hours. All adverse events were recorded.

Results: 19 patients were randomized, 8 received exenatide. An interim recruitment failure analysis with subsequent changes of the protocol was made. The study was stopped prematurely due to slow inclusion. No difference was observed in the main outcome of plasma glucose at $4 \mathrm{~h}$; control vs. exenatide [mean, $S D](7.0 \pm 1.9$ vs. $7.6 \pm 1.6 ; \mathrm{p}=0.56)$. No major adverse events were reported.

Conclusions: We found no evidence that prehospital exenatide had effect on hyperglycemia. However, it was given without adverse events in this study with limited sample size that was prematurely stopped due to slow inclusion.

Clinical Trial Registration: "PROLOGUES - Prehospital lowering of glucose in Stroke”, URL: www.clinicaltrialsregister.eu (2011-002780-16).

Keywords: Emergency medical services, ambulances, stroke, diabetes mellitus, glucagonlike peptide 1 , hyperglycemia. 


\section{Introduction}

Stroke, of which ischemic stroke account for around $80 \%$, is the second most common cause of mortality and the third most common cause of disability. ${ }^{1,2}$ Diabetes is a well-established risk factor for stroke incidence, poor outcome after stroke, and risk for recurrent stroke. ${ }^{3-5}$ Approximately one third of patients suffering an ischemic stroke have diabetes in international studies. ${ }^{5}$ In the national Swedish Stroke Registry the numbers are somewhat lower with $22 \%$ of patients having diabetes. ${ }^{6}$

Admission hyperglycemia is, independently from diabetes, a negative prognostic marker for patients suffering acute ischemic stroke. Interestingly, hyperglycemia in patients without previously known diabetes is associated with a greater risk for poor outcome compared to patients with identified diabetes prior the stroke..$^{5,7}$ Trials addressing hyperglycemia with early onset insulin treatment have, however, failed to show any benefit on the outcome of stroke. ${ }^{9,10}$ Additionally, several of these trials report a high incidence of hypoglycemia. ${ }^{11}$

Glucagon-like peptide-1 receptor agonists (GLP-1RA) is a class of antihyperglycemic drugs that reduce glucose in a glucose-dependent manner with a minimal risk for hypoglycemia. ${ }^{12-14}$ Epidemiologic studies have shown that treatment with GLP-1RA reduces the risk for major cardiovascular events and large randomized clinical trials have confirmed this finding. ${ }^{15-20}$ There is a growing body of evidence from preclinical studies using experimental stroke models that GLP-1RA treatment can also reduce stroke-induced brain damage both under hyper- and normoglycemic conditions. ${ }^{21-26}$ Most studies have used pre-treatment, but effect has also been shown with administration after stroke. ${ }^{22}$ It is undetermined whether treatment with GLP-1RA can affect the clinical outcome after ischemic stroke in humans. ${ }^{27}$

\section{Aims}

To date there are no clinical trials that have reported the efficacy and feasibility of GLP-1RA prehospital administration on patients admitted to hospital for stroke.

The triad of antihyperglycemic effects, minimal risk of hypoglycemia and neuroprotection makes GLP-1RA an extremely interesting treatment option in patients 
exposed to ischemic stroke and hyperglycemia. We therefore designed this study evaluating safety and feasibility of treatment with the GLP-1RA exenatide in a prehospital setting. The primary endpoint was a reduction in plasma glucose of $2.0 \mathrm{mmol} / \mathrm{L}$ after 4 hours. The secondary endpoint was safety of prehospital administration of exenatide in acute stroke (adverse events e.g. hypoglycemic episodes or problems reported by the ambulance nurse).

\section{Materials \& Methods}

\section{Trial design and oversight}

The study was an open label randomized controlled trial comparing prehospital exenatide treatment started in ambulance compared to standard care (i.e. insulin treatment at hospital). The study was approved by the Central Ethics Committee of Sweden (Ref. No. Ö 62012). Written informed consent was obtained in the ambulance from all participants. Patients were randomized in a 1:1 ratio in randomization blocks of 4 . The decentralized organization of the ambulance service ensured that the ambulance nurses had no knowledge about previous randomization results. The study center was Södersjukhuset in Stockholm, Sweden: a large urban teaching hospital that treats approximately 1000 patients every year for ischemic stroke out of which around $75 \%$ arrive by ambulance. The protocol was published prior to the start of the trial on clinicaltrialsregister.eu (EudraCT number: 2011002780-16). Two changes to the protocol was added after start of study: 1) widening of glucose inclusion criteria from 10-15 mmol/L to first 9-15 mmol/L and then 8-15 mmol/L and 2) inclusion of patients with previously known metformin treated type 2 diabetes. These changes were made due to slow recruitment.

\section{Patients}

Patients transported to hospital by ambulance with symptoms of stroke and hyperglycemia were considered eligible for the study. Inclusion criteria was: $\geq 1$ point on the FAST-test ${ }^{28}$ (Face Arm Speech Time), symptoms of stroke with a duration less than 6 hours, capillary plasma glucose 8-15 mmol/L, age $\geq 18$ and signed informed consent. Exclusion criteria were: type 1 diabetes, antidiabetic treatment other than metformin, pregnancy, cirrhotic liver disease, regular hemo- or peritoneal dialysis, GCS $<14$ or GCS verbal $<5$, signs of pharyngeal palsy or previously known dementia. 


\section{Procedures and outcomes}

Patients were screened and included by the registered prehospital nurse. 90 nurses were trained and individually delegated to include patients. Study physician (ML) was on call around the clock for consultations throughout the study period. Patients randomized to exenatide received a single subcutaneous injection of $10 \mu \mathrm{g}$ exenatide in the ambulance enroute to hospital. Patients randomized to standard care received no study treatment in the ambulance. Patients were kept fasting the first 4 hours to achieve stable and comparable conditions. Venous samples were collected before treatment and after 4 and 24 hours. Capillary glucose measurements were made every hour for the first 4 hours, then every 4 hours until end of study. Total study period was 24 hours. Insulin on a sliding scale was used for comparator, except for the first 4 hours. Insulin could, however, be administered at any time by the treating physician if considered clinically important. Adverse events and insulin use was recorded in the case report form.

To achieve reliable and comparable capillary measurements a high precision glucometer, Accu-Chek ${ }^{\circledR}$ Inform II (Roche Diagnostics, Rotkreuz, Switzerland) was chosen as primary glucometer in the study, this glucometer also allowed for automated data transfer to a server. ${ }^{29}$ In case the Accu-Chek ${ }^{\circledR}$ Inform II was non-operational the regular glucometer at the point of care was used instead. Venous plasma glucose was measured at a core lab facility, The Karolinska University laboratory, with the hexokinase method on $\operatorname{cobas}^{\circledR} 8000$ (c701), Roche Diagnostics.

Primary outcome was a reduction of plasma glucose with $2.0 \mathrm{mmol} / \mathrm{L}$ at 4 hours after randomization. Secondary outcomes were: overall feasibility of prehospital treatment (adverse events and problems reported by the ambulance nurses), and as an exploratory post hoc outcome the AUC of glucose during 4 and 24 hours.

\section{Statistical analysis}

Power calculation gave a sample size of 42 to detect a $2 \mathrm{mmol} / \mathrm{L}$ difference in plasma glucose at 4 hours with a power of $90 \%$ and a two-sided alpha level of 0.05 .

The differences in 4 hour P-glucose, AUC of glucose during the first 4 hours and during the total study period of 24 hours were analyzed with Mann-Whitney U-test. AUC of glucose was calculated with the trapezoid formula. Missing data on capillary glucose for the AUC calculations was handled with imputation of mean value of adjacent time points. 
Individuals with $\leq 40 \%$ valid measurements were excluded $(n=2,1$ exenatide and 1 control). An additional analysis was made with linear regression adjusting for sex, age and BMI. Analyses presented are made according to Intention To Treat (ITT)

SPSS v23.0.0.3 (IBM Corp, Armonk, NY, USA) was used for statistical analysis. A two sided p-value $<0,05$ was considered statistically significant.

\section{Results}

\section{Overview of study}

The study was open for inclusion between May 2013 and May 2018 and was stopped due to slow inclusion. A total of 19 patients were included in the study, 8 received exenatide. One patient was transferred to a tertiary center for thrombectomy. That record was obtained retrospectively and evaluated for outcomes and adverse events. Baseline characteristics are shown in table 1.

\section{Recruitment failure analysis}

An analysis of recruitment failure was made in 2015 due to slow inclusion. The largest participating ambulance station responsible for approximately $25 \%$ of the ambulance transports to the study hospital and $50 \%$ of the study ambulances was reviewed. Among the non-included patients during the first four months of 2015 a total of 143 dispatches were made for suspected stroke. The suspicion remained in 69 patients after initial ambulance nurse evaluation. Out of them 13 patients had a glucose value of 9-15 mmol/L, which was the inclusion range at time of review. Two of these patients met all criteria and could have been included and was as such true recruitment failures.

The remaining eleven patients would have been excluded mainly due to problems with obtaining informed consent; three were confused, two had reduced level of consciousness, one had hearing impairment and one needed morphine for pain control. Three patients did not meet the inclusion criteria and one was deemed non suitable by the ambulance nurse (ambulance transit time 3 minutes).

With a glucose inclusion interval of 8-15 $\mathrm{mmol} / \mathrm{L}$ the 13 patients would have increased to 19. As a result of this analysis the glucose interval for inclusion was expanded to $8-15 \mathrm{mmol} / \mathrm{L}$. 


\section{Glucose outcomes}

There was no difference between the exenatide treated group and the control group in pglucose levels at 4 hours (7.6 vs. 7.0, $\mathrm{P}=0.56)$, Table 2. Total glucose exposure measured with AUC showed no difference: AUC 0-4h (33.7 vs. 32.9, P=0.93) or AUC 0-24h (186.8 vs. 184.3, $\mathrm{P}=0.90$ ), Figure 1.

On the main outcome variable, venous p-glucose at 4 hours, data was missing for 3 individuals ( 2 exenatide, 1 control). The primary analysis is without imputation. A sensitivity analysis with the capillary values imputed for venous did not change the results significantly. Linear regression of venous glucose at 4 hours with adjustment for age, sex and BMI resulted in similar results (exenatide vs. control: 7.1 vs. $7.4, \mathrm{P}=0.76$ ). Sensitivity analyses for AUC-measures with either the highest or lowest adjacent values imputed were made, but did not alter the results.

Analyses including only individuals with stroke diagnoses at discharge were performed for all analyses but did not alter the results significantly (data not shown).

\section{Insulin treatment}

No insulin was administrated during the study period as the criterion for insulin treatment was not met for any individual.

\section{Adverse events}

No major adverse events were observed during the study period. One patient in the exenatide group suffered from nausea and vomiting. However, that participant suffered from basilar thrombosis and had vomited before inclusion. No patients experienced hypoglycemia.

\section{Discussion}

Preclinical studies showing favorable effects of GLP-1RA in stroke encourage clinical studies of these diabetes drugs for stroke treatment. ${ }^{27}$ However, the clinical efficacy of acute neuroprotection after stroke is largely dependent on timely intervention within a very short therapeutic window to minimize stroke-induced brain loss (few hours from stroke onset), which is very difficult to achieve. To gain new knowledge in this field, this study was designed as a pilot to investigate whether it was possible and safe to administer exenatide in a prehospital setting and lower plasma glucose. Our study found no evidence that 
prehospital exenatide lowered glucose levels in acute stroke, but that it was feasible to perform without any serious adverse events or hypoglycemia. However, as the study was prematurely closed due to slow inclusion, it also shows the difficulty of this type of study.

Both the exenatide and the control groups were hyperglycemic in the first hours post stroke onset, but the glucose level dropped equally. The initial hyperglycemia may have been caused by stress hyperglycemia, which is known to be present after stroke.,11

The exenatide group also had higher NIHSS-score compared with the control group (median 4.5 vs. 1). Larger strokes could potentially produce higher stress levels and cause a more severe stress hyperglycemia that could mask out a potential treatment effect. Some patients only had moderate levels of hyperglycemia as the inclusion threshold was 8 $\mathrm{mmol} / \mathrm{L}$ making detection of glucose reduction harder. The statistical phenomenon "regression to mean" could also play a role as all patients included were selected on the basis of an abnormal lab value, i.e. hyperglycemia.

Another potential reason for low effect we cannot rule out is decreased bioactivity of exenatide in the ambulances since storage of the drug was in the ambulances with potentially varying temperatures. However, the prefilled exenatide syringes were exchanged continuously before expiration date throughout the study period and were stored in an insulating casein the ambulances. Considering both that the study drug was continuously exchanged and was stored in a protected manner in the ambulances we consider the risk of significant degradation of exenatide to be low.

One of the most common side effects in clinical use of GLP-1RA is nausea and vomiting. ${ }^{30,31}$ In our study one patient experienced these symptoms. That patient suffered from a basilar thrombosis and was vomiting before randomization to exenatide. Hence, no patients in our study experienced GLP-1RA induced nausea. Vomiting from clinical use of GLP-1RA could in part be mediated by slowed gastric emptying. ${ }^{32}$ In the acute phase of stroke nil per os is often ordered before oropharyngeal function is known, simultaneously reducing this potential problem of GLP-1RA side effects. As our study demonstrated that exenatide can be administrated in this vulnerable group of patients with very few reported adverse events including nausea and vomiting, we suggest that GLP-1RA agonists is a safe potential treatment option on stroke patients even with potential pharyngeal palsy. 
Our study was stopped early due to slow inclusion. As it is of paramount importance that study subjects understand the concept of the study and can give an informed consent to participate, the present study in a study-population with high risk for cognitive impairment and aphasia present a great challenge in this regard. Therefore a large number of patients with aphasia and suspected cognitive dysfunction were excluded from participation in the study. This was also shown by our recruitment failure analysis where only 13 of 143 cases of suspected stroke remained with good fit for the study. Moreover, the importance of reducing time to rtPA treatment may have stressed the ambulance nurses to not include potential study subjects in the prehospital setting. The most important reason for not being eligible for inclusion was, however, the glucose range. Even mild hyperglycemia was relatively uncommon with 13 of 69 patients having the stipulated glucose range of 9-15 mmol/L. This resulted in an amendment to the protocol to expand the range to 8-15 mmol/L. Another obstacle to maintaining inclusion was the turnover of nurses working at the participating stations. Because inclusion in the ambulance could only be made by a nurse delegated to do so, every time a nurse changed employer a new one had to be trained and delegated or potential patients would be missed. In total 90 nurses received study training and individual delegation.

There are several limitations of the study. The most important shortcoming of the study is the low number of included patients. The limited sample size warrants some caution in interpretation of the lack of adverse events as a lower frequency than $35 \%$ cannot be expected to be detected. ${ }^{33}$ Another limitation is the short follow-up of 24 hours. Finally, as inclusion in the study was prehospital some of the included patients were found to have other discharge diagnoses than stroke.

However, the real world situation for ambulance nurses is one of uncertainty with a lack of background information, labs and no x-ray. This makes transfer of protocols developed in a hospital arena to the prehospital setting hard. The fact that our study was performed in a real prehospital setting without adverse events is promising, but the limited study sizes makes it impossible to draw definitive conclusions about safety.

Our study could not, and was not designed to, determine if early treatment with GLP-1RA could reduce poor outcome after stroke. Importantly, GLP-1RA have been shown to be neuroprotective in animal studies under normoglycemic conditions even with treatment 
initiated early after stroke. ${ }^{22}$ Therefore, larger studies evaluating other GLP-1RA than exenatide with a focus on clinical outcomes after stroke should be launched in a near future. ${ }^{27}$

\section{Conclusion}

The present study found no evidence to suggest that prehospital treatment with exenatide on patients with stroke symptoms lowers preexisting mild hyperglycemia. On the other hand, administration of exenatide was not associated with adverse events or other problems. The study was stopped prematurely due to slow recruitment of patients that was caused by narrow inclusion and exclusion criteria in combination with the difficulties posed by including patients with stroke in a prehospital environment.

\section{References}

1. Feigin VL, Norrving B, Mensah GA. Global Burden of Stroke. Circ Res. 2017;120(3):439-448.

2. Krishnamurthi RV, Feigin VL, Forouzanfar MH, et al. Global and regional burden of first-ever ischaemic and haemorrhagic stroke during 1990-2010: findings from the Global Burden of Disease Study 2010. The Lancet Global Health. 2013;1(5):e259-e281.

3. Rautio A, Eliasson M, Stegmayr B. Favorable trends in the incidence and outcome in stroke in nondiabetic and diabetic subjects: findings from the Northern Sweden MONICA Stroke Registry in 1985 to 2003. Stroke; a journal of cerebral circulation. 2008;39(12):3137-3144.

4. Kannel WB, McGee DL. Diabetes and cardiovascular risk factors: the Framingham study. Circulation. 1979;59(1):8-13.

5. Lau LH, Lew J, Borschmann K, Thijs V, Ekinci EI. Prevalence of diabetes and its effects on stroke outcomes: A meta-analysis and literature review. J Diabetes Investig. 2018.

6. Riksstroke. Stroke och TIA Riksstrokes årsrapport 2017. http://www.riksstroke.org2018.

7. Capes SE, Hunt D, Malmberg K, Pathak P, Gerstein HC. Stress hyperglycemia and prognosis of stroke in nondiabetic and diabetic patients: a systematic overview. Stroke; a journal of cerebral circulation. 2001;32(10):2426-2432. 
8. Weir CJ, Murray GD, Dyker AG, Lees KR. Is hyperglycaemia an independent predictor of poor outcome after acute stroke? Results of a long term follow up study. Bmj. 1997;314(7090):1303.

9. Gray CS, Hildreth AJ, Sandercock PA, et al. Glucose-potassium-insulin infusions in the management of post-stroke hyperglycaemia: the UK Glucose Insulin in Stroke Trial (GIST-UK). Lancet neurology. 2007;6(5):397-406.

10. Rosso C, Corvol JC, Pires C, et al. Intensive versus subcutaneous insulin in patients with hyperacute stroke: results from the randomized INSULINFARCT trial. Stroke; $a$ journal of cerebral circulation. 2012;43(9):2343-2349.

11. Kruyt ND, Biessels GJ, Devries JH, Roos YB. Hyperglycemia in acute ischemic stroke: pathophysiology and clinical management. Nature reviews Neurology. 2010;6(3):145155.

12. Holz Iv GG, Kiihtreiber WM, Habener JF. Pancreatic beta-cells are rendered glucosecompetent by the insulinotropic hormone glucagon-like peptide-1(7-37). Nature. 1993;361(6410):362-365.

13. Gutniak M, Ørkov C, Holst JJ, Ahrén B, Efendić S. Antidiabetogenic Effect of Glucagonlike Peptide-1 (7-36)amide in Normal Subjects and Patients with Diabetes Mellitus. New England Journal of Medicine. 1992;326(20):1316-1322.

14. Gutniak MK, Linde B, Holst JJ, Efendic S. Subcutaneous injection of the incretin hormone glucagon-like peptide 1 abolishes postprandial glycemia in NIDDM. Diabetes Care. 1994;17(9):1039-1044.

15. Zimmerman RS, Hobbs TM, Wells BJ, et al. Association of glucagon-like peptide-1 receptor agonist use and rates of acute myocardial infarction, stroke and overall mortality in patients with type 2 diabetes mellitus in a large integrated health system. Diabetes, obesity \& metabolism. 2017;19(11):1555-1561.

16. Best JH, Hoogwerf BJ, Herman WH, et al. Risk of cardiovascular disease events in patients with type 2 diabetes prescribed the glucagon-like peptide 1 (GLP-1) receptor agonist exenatide twice daily or other glucose-lowering therapies: a retrospective analysis of the LifeLink database. Diabetes Care. 2011;34(1):90-95.

17. Paul SK, Klein K, Maggs D, Best JH. The association of the treatment with glucagonlike peptide-1 receptor agonist exenatide or insulin with cardiovascular outcomes in 
patients with type 2 diabetes: a retrospective observational study. Cardiovasc Diabetol. 2015;14:10.

18. Marso SP, Bain SC, Consoli A, et al. Semaglutide and Cardiovascular Outcomes in Patients with Type 2 Diabetes. The New England journal of medicine. 2016;375(19):1834-1844.

19. Marso SP, Daniels GH, Brown-Frandsen K, et al. Liraglutide and Cardiovascular Outcomes in Type 2 Diabetes. The New England journal of medicine. 2016;375(4):311322.

20. Hernandez AF, Green JB, Janmohamed S, et al. Albiglutide and cardiovascular outcomes in patients with type 2 diabetes and cardiovascular disease (Harmony Outcomes): a double-blind, randomised placebo-controlled trial. Lancet. 2018;392(10157):1519-1529.

21. Marlet IR, Olmestig JNE, Vilsboll T, Rungby J, Kruuse C. Neuroprotective Mechanisms of Glucagon-like Peptide-1-based Therapies in Ischaemic Stroke: A Systematic Review based on Pre-Clinical Studies. Basic \& clinical pharmacology \& toxicology. 2018;122(6):559-569.

22. Darsalia V, Hua S, Larsson $M$, et al. Exendin-4 reduces ischemic brain injury in normal and aged type 2 diabetic mice and promotes microglial M2 polarization. PloS one. 2014;9(8):e103114.

23. Darsalia V, Mansouri S, Ortsater $\mathrm{H}$, et al. Glucagon-like peptide-1 receptor activation reduces ischaemic brain damage following stroke in Type 2 diabetic rats. Clin Sci (Lond). 2012;122(10):473-483.

24. Teramoto S, Miyamoto N, Yatomi K, et al. Exendin-4, a glucagon-like peptide-1 receptor agonist, provides neuroprotection in mice transient focal cerebral ischemia. Journal of cerebral blood flow and metabolism : official journal of the International Society of Cerebral Blood Flow and Metabolism. 2011;31(8):1696-1705.

25. Darsalia V, Klein T, Nystrom T, Patrone C. Glucagon-like receptor 1 agonists and DPP4 inhibitors: Anti-diabetic drugs with anti-stroke potential. Neuropharmacology. 2017.

26. Filchenko I, Simanenkova A, Chefu S, Kolpakova M, Vlasov T. Neuroprotective effect of glucagon-like peptide-1 receptor agonist is independent of glycaemia normalization 
in type two diabetic rats. Diabetes and Vascular Disease Research. 2018;15(6):567570.

27. Darsalia V, Larsson M, Klein T, Patrone C. The high need for trials assessing functional outcome after stroke rather than stroke prevention with GLP-1 agonists and DPP-4 inhibitors. Cardiovasc Diabetol. 2018;17(1):32.

28. Nor AM, McAllister C, Louw SJ, et al. Agreement between ambulance paramedic- and physician-recorded neurological signs with Face Arm Speech Test (FAST) in acute stroke patients. Stroke; a journal of cerebral circulation. 2004;35(6):1355-1359.

29. Jeong TD, Cho EJ, Ko DH, et al. Large-scale performance evaluation of Accu-Chek inform II point-of-care glucose meters. Scand J Clin Lab Invest. 2016;76(8):657-663.

30. Consoli A, Formoso G. Potential side effects to GLP-1 agonists: understanding their safety and tolerability. Expert Opin Drug Saf. 2015;14(2):207-218.

31. Htike ZZ, Zaccardi F, Papamargaritis D, Webb DR, Khunti K, Davies MJ. Efficacy and safety of glucagon-like peptide- 1 receptor agonists in type 2 diabetes: A systematic review and mixed-treatment comparison analysis. Diabetes, obesity \& metabolism. 2017;19(4):524-536.

32. Jelsing J, Vrang N, Hansen G, Raun K, Tang-Christensen M, Knudsen LB. Liraglutide: short-lived effect on gastric emptying -- long lasting effects on body weight. Diabetes, obesity \& metabolism. 2012;14(6):531-538.

33. Onakpoya IJ. Rare adverse events in clinical trials: understanding the rule of three. BMJ Evid Based Med. 2018;23(1):6.

COBAS and ACCU-CHEK are trademarks of Roche. 


\section{Tables}

Table 1. Baseline characteristics of study participants (Control N=11, Exenatide $\mathrm{N}=8$ )

\begin{tabular}{|l|l|l|}
\hline & Control (IQR) & Exenatide (IQR) \\
\hline Age, years & $80(63-89)$ & $71(54-82)$ \\
\hline Male sex, $\mathrm{n}(\%)$ & $6(55)$ & $4(50)$ \\
\hline BMI, kg/m & $26.4(23.2-31.7)$ & $27.2(21.6-29.5)$ \\
\hline $\begin{array}{l}\text { vP-Glucose, mmol/L } \\
\text { Systolic blood pressure, } \\
\text { mmHg }\end{array}$ & $9.6(9.0-10.8)$ & $9.6(8.4-10.6)$ \\
\hline $\begin{array}{l}\text { Diastolic blood pressure, } \\
\text { mmHg }\end{array}$ & $89(80-100)$ & $8.8(6.9-10.9)$ \\
\hline Heart rate & $88(72-9-11.2)$ & $150(124-175)$ \\
\hline NIHSS & $1(0-4)$ & $80(68-89)$ \\
\hline Discharge diagnosis & & $4.5(1-8)$ \\
\hline Ischemic Stroke, $\mathrm{n}$ & 9 & 4 \\
\hline Hemorrhagic Stroke, $\mathrm{n}$ & 0 & 2 \\
\hline Non-Stroke, $\mathrm{n}$ & 2 & 2 \\
\hline Treated with rtPA & 1 & 0 \\
\hline Data is & & \\
\hline
\end{tabular}

Data is presented as median with interquartile range unless otherwise specified.

$\mathrm{IQR}=$ interquartile range, $\mathrm{BMI}=$ body mass index, $\mathrm{cP}$-Glucose = capillary plasma glucose, $\mathrm{vP}-$

Glucose $=$ venous plasma glucose, NIHSS $=$ National Institutes of Health Stroke Scale, $r$ PA $=$ recombinant tissue Plasminogen Activator.

Table 2. Outcome measures (Control $\mathrm{N}=11$, Exenatide $\mathrm{N}=8$ )

\begin{tabular}{|l|l|l|l|}
\hline & Control \pm SD & Exenatide \pm SD & P \\
\hline $\begin{array}{l}\text { vP-Glucose } 4 \mathrm{~h}, \\
\mathrm{mmol} / \mathrm{L}\end{array}$ & $7.0 \pm 1.9$ & $7.6 \pm 1.6$ & 0.56 \\
\hline
\end{tabular}




\begin{tabular}{|l|l|l|l|}
\hline cP-Glucose $4 \mathrm{~h}, \mathrm{mmol} / \mathrm{L}$ & $6.5 \pm 1.5$ & $7.3 \pm 2.0$ & 0.62 \\
\hline AUC 0-4 h, glu x h & $32.9 \pm 6.2$ & $33.7 \pm 9.6$ & 0.93 \\
\hline AUC 0-24h, glu x h & $184.3 \pm 41$ & $186.8 \pm 40$ & 0.90 \\
\hline Adverse events, $\mathrm{n}$ & 0 & $1^{\dagger}$ & \\
\hline Need of insulin, $\mathrm{n}$ & 0 & 0 & \\
\hline
\end{tabular}

${ }^{\dagger}$ Nausea and vomiting first $24 \mathrm{~h}$, patient suffered from basilar thrombosis. Data presented as means \pm standard deviation. $\mathrm{vP}-$ Glucose $=$ venous plasma glucose, $\mathrm{cP}-$ Glucose $=$ capillary plasma glucose. Variables tested with Mann-Whitney U-test. 


\section{Figure Legends}

Figure 1. Capillary plasma glucose. P-values in figure represent Mann-Whitney U-test of AUC 0-4h and 0-24h. Bars indicate standard deviation. 


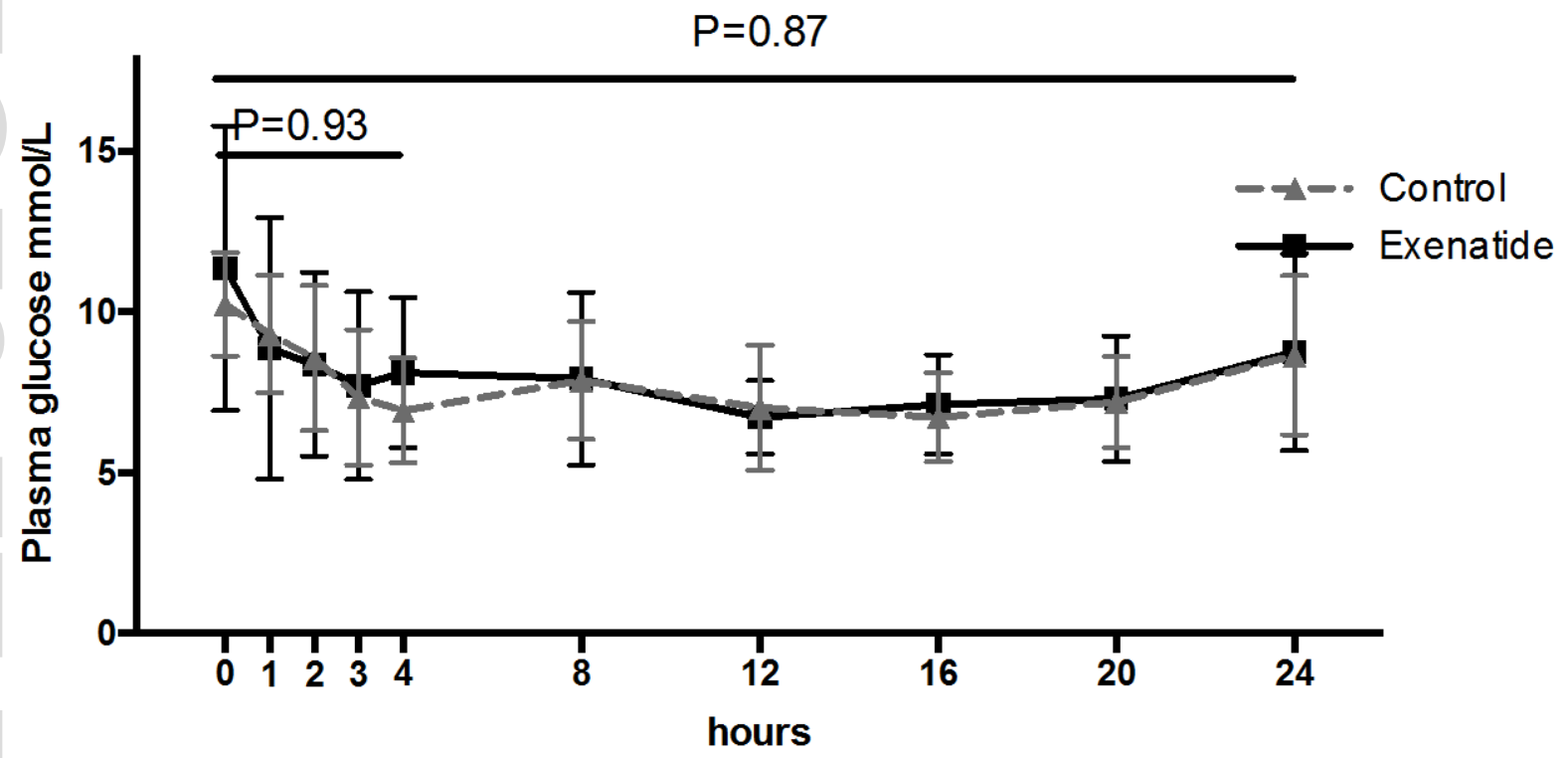

Figure 1. Capillary plasma glucose. P-values in figure represent Mann-Whitney U-test of AUC 0-4h and 0-24h. Bars indicate standard deviation. 